\title{
A NOTE ON AN INTEGRAL INEQUALITY ${ }^{1}$
}

\author{
PAUL R. BEESACK
}

Recently, K. Tatarkiewicz [1] proved an interesting integral inequality which we shall state as Theorem I. The purpose of this note is to prove-by precisely the same technique used in [1]-a considerably more general result (Theorem II). We finally apply Theorem II to the proof of a result comparing the first eigenvalues of two second-order, linear homogeneous differential systems (Theorem III).

Theorem I (Tatarkiewicz). Let $A_{1}, A_{2}$ be two measurable sets. Let $f$ be a function measurable on $A=A_{1} \cup A_{2}$, and let $g$ be a function integrable on $A$. If

$$
0 \leqq f\left(p_{1}\right) \leqq g\left(p_{1}\right) \leqq g\left(p_{2}\right) \leqq f\left(p_{2}\right)
$$

for every pair $p_{1}, p_{2}$ of points such that $p_{1} \in A_{1}, p_{2} \in A_{2}$, and if

$$
\int_{A} g(p) d p \leqq \int_{A} f(p) d p
$$

then

$$
\int_{A}[g(p)]^{2} d p \leqq \int_{A}[f(p)]^{2} d p .
$$

As a corollary of Theorem II, we shall prove that (3) may be replaced $^{2}$ by

$$
\int_{A}[g(p)]^{n} d p \leqq \int_{A}[f(p)]^{n} d p, \quad n=2,3, \cdots,
$$

in case both hypotheses (1) and (2) are retained. On the other hand, we will see that (3) remains valid if (1) is replaced by either of the conditions

Presented to the Society, October 27, 1956; received by the editors September 14, 1956 and, in revised form, January 4, 1957.

1 This note was written while the author held a Summer Research Associateship (1956), of the National Research Council of Canada.

2 Under the additional assumption that $f$ is also integrable over $A$. See remarks following Theorem II. The referee has pointed out that hypotheses (1) and (2) actually imply not just (4), but $\int_{\phi}[g(p)] d p \leqq \int_{\phi}[f(p)] d p$ for any continuous function $\phi(x)$ which is convex and monotone increasing for $x \geqq 0$. This follows, not from Theorem II - which is a generalization of a different nature-but from a result in Hardy, Littlewood and Pólya, Inequalities, 1934, p. 170, Theorem 249. 
(5 $\left.5_{1}\right) \quad f\left(p_{1}\right) \leqq g\left(p_{1}\right), \quad 0 \leqq f\left(p_{1}\right)+g\left(p_{1}\right) \leqq f\left(p_{2}\right)+g\left(p_{2}\right), \quad g\left(p_{2}\right)<f\left(p_{2}\right) ;$

$\left(5_{2}\right) \quad f\left(p_{1}\right) \leqq g\left(p_{1}\right), \quad f\left(p_{1}\right)+g\left(p_{1}\right) \leqq 0 \leqq f\left(p_{2}\right)+g\left(p_{2}\right), \quad g\left(p_{2}\right)<f\left(p_{2}\right)$.

In particular, $f$ and $g$ need not be non-negative, as required by (1). Before proceeding with the proof of Theorem II, we give two examples illustrating (4) and $\left(5_{1}\right)$.

ExAmple 1 . Let $A_{1}, A_{2}$ be the intervals $0 \leqq x \leqq 1 / 2,1 / 2<x \leqq 1$ respectively, and let $f(x)=(5 / 2) x-1, g(x)=(-3 / 2) x+1$. Then $f(x)$ $+g(x) \equiv x$ so that $\left(5_{1}\right)$ is satisfied. Moreover,

$$
\int_{0}^{1} g(x) d x=\frac{1}{4}=\int_{0}^{1} f(x) d x
$$

so that (2) is satisfied. We conclude that $\int_{0}^{1} g^{2}(x) d x \leqq \int_{0}^{1} f^{2}(x) d x$. Hypothesis (1), of course, is not satisfied.

Example 2. Let $A_{1}, A_{2}$ be the intervals $\pi / 4 \leqq x \leqq \pi / 2,0 \leqq x<\pi / 4$ respectively, and let $f(x)=\cos ^{2} x, g(x)=1-(2 x / \pi)$. One easily verifies that condition (1) is satisfied, while both integrals in (2) have the value $\pi / 4$. According to (4) we have

$$
\frac{\pi}{2(n+1)}=\int_{0}^{\pi / 2}\left(1-\frac{2 x}{\pi}\right)^{n} d x \leqq \int_{0}^{\pi / 2} \cos ^{2 n} x d x=\frac{(2 n) !}{2^{2 n}(n !)^{2}} \cdot \frac{\pi}{2},
$$

which, incidentally, gives the lower bound

$$
\frac{1}{n+1} \leqq \frac{1 \cdot 3 \cdots(2 n-1)}{2 \cdot 4 \cdots 2 n}, \quad n=2,3, \cdots .
$$

It is of interest to note that-in this case-if $g(x)$ is replaced by $2 x / \pi$, the inequality (4) clearly does not change. However, (1) is no longer satisfied, whereas $\left(5_{1}\right)$ is now satisfied. In general, $\left(5_{1}\right)$ is not sufficient to imply (4) for $n>2$, so that we could not draw this (true) conclusion from (1) or $\left(5_{1}\right)$.

Theorem II. Let $F(x), G(x), M(x)$ be integrable functions over a measurable set $A$. Let

$$
A_{1}=\{x: F(x) \leqq G(x)\}, \quad A_{2}=\{x: F(x)>G(x)\},
$$

and suppose that

$$
\int_{A} G(x) d x \leqq \int_{A} F(x) d x,
$$

and either of the conditions

$$
\begin{aligned}
0 & \leqq M\left(x_{1}\right) \leqq M\left(x_{2}\right) . \\
M\left(x_{1}\right) & \leqq 0 \leqq M\left(x_{2}\right),
\end{aligned}
$$


is satisfied for every pair $x_{1}, x_{2}$ of points such that $x_{1} \in A_{1}, x_{2} \in A_{2}$. Then

$$
\int_{\boldsymbol{A}} G(x) M(x) d x \leqq \int_{\boldsymbol{A}} F(x) M(x) d x .
$$

Proof. Since

$$
\int_{A_{1}} G(x) d x+\int_{A_{2}} G(x) d x \leqq \int_{A_{1}} F(x) d x+\int_{A_{2}} F(x) d x
$$

it follows that

$$
\int_{A_{1}}(G-F) d x \leqq-\int_{A_{2}}(G-F) d x .
$$

If now, we define the function $H(x)=G(x)-F(x)$, then $H(x) \geqq 0$, $x \in A_{1}$, while $H(x)<0, x \in A_{2}$. Moreover, $H(x)$ is integrable over $A$, and

$$
\int_{A_{1}} H d x \leqq-\int_{A_{2}} H d x
$$

Suppose that $\left(7_{1}\right)$ is satisfied. Then there exists a constant $c \geqq 0$ such that

$$
0 \leqq M\left(x_{1}\right) \leqq c \leqq M\left(x_{2}\right), \quad x_{1} \in A_{1}, x_{2} \in A_{2} .
$$

We now have

$$
\begin{array}{ll}
0 \leqq H(x) M(x) \leqq c H(x), & x \in A_{1}, \\
H(x) M(x) \leqq c H(x) \leqq 0, & x \in A_{2} .
\end{array}
$$

Using (9), it now follows that

$$
\int_{A_{1}} H(x) M(x) d x \leqq c \int_{A_{1}} H(x) d x \leqq-c \int_{A_{2}} H(x) d x,
$$

and

$$
\int_{A_{2}} H(x) M(x) d x \leqq c \int_{A_{2}} H(x) d x .
$$

Adding inequalities, we now obtain

$$
\int_{A} H(x) M(x) d x \leqq 0,
$$

which is equivalent to (8).

Finally, if $\left(7_{2}\right)$ is satisfied, then we have $M(x) H(x) \leqq 0$ for all $x \in A$, whence (10) again follows. 
Since (1) implies $\left(5_{1}\right)$ we may show that Theorem II (essentially) includes Theorem I by proving that (2) and ( $\left.5_{1}\right)$ imply (3). Indeed, (assuming that $f(x)$ is also integrable over $A$ ), the functions $F(x) \equiv 0$, $G(x)=g(x)-f(x), M(x)=g(x)+f(x)$ are also integrable. The sets $A_{1}$, $A_{2}$ of Theorem II are just

$$
A_{1}=\{x: f(x) \leqq g(x)\}, \quad A_{2}=\{x: g(x)<f(x)\},
$$

while condition $\left(5_{1}\right)$ is just $\left(7_{1}\right)$, and (2) implies (6). Hence, by Theorem II, we conclude

$$
\int_{A}(g-f)(g+f) d x \leqq \int_{A} 0 \cdot(g+f) d x=0,
$$

and thus (3). Similarly, (2) and ( 52 imply (3). The form (11) of conclusion (3) shows why we require the additional hypothesis that $f(x)$ is also integrable. For, the conditions of Theorem I imply the existence-finite or $+\infty$ - of all the integrals appearing in that theorem. On the other hand, the integral appearing in the left side of (11) may not exist under these hypotheses.

In order to deduce conclusion (4) from (1) and (2), we set $F(x) \equiv 0$, $G(x)=g(x)-f(x), \quad M(x)=g^{n-1}+g^{n-2} f+\cdots+f^{n-1}$, and the result follows as before. Of course, for specified $n$, (1) may be replaced by weaker hypotheses analogous to (5).

THEOREM III. Let $p(x), q(x)$ be continuous on $-a \leqq x \leqq a$, and symmetric about $x=0$. Suppose that $p(x)$ is non-negative on this interval, and that

$$
\int_{-a}^{a} q(x) d x=\int_{-a}^{a} p(x) d x .
$$

Consider the following differential systems

$$
\begin{aligned}
u^{\prime \prime}(x)+\lambda_{p} p(x) u(x) & =0, & & u( \pm a)=0, \\
v^{\prime \prime}(x)+\lambda_{q} q(x) v(x) & =0, & & v( \pm a)=0 .
\end{aligned}
$$

Let $\lambda_{p}$ and $\lambda_{q}$ also denote the least positive eigenvalues of (13) and (14) respectively. (So we assume $q(x)>0$ for some points of $-a \leqq x \leqq a$.) If there exists $a$ point $b, 0<b<a$ such that

$$
q(x) \geqq p(x), \quad 0 \leqq x \leqq b, \text { and } q(x) \leqq p(x), \quad b \leqq x \leqq a,
$$

then $\lambda_{q} \leqq \lambda_{p}$. Moreover, strict inequality holds unless $p(x) \equiv q(x)$.

Proof. Let $u(x)$ be an eigenfunction corresponding to the eigenvalue $\lambda_{p}$. Then $u(x)$ may be taken to be positive and symmetrically 
decreasing about $x=0$ on $-a<x<a$. In Theorem II, set $F(x)=q(x)$, $G(x)=p(x), M(x)=[u(x)]^{2}$. Then, by (15)

$$
A_{1}=\{x: b \leqq|x| \leqq a\}, \quad A_{2}=\{x: 0 \leqq|x|<b\},
$$

while

$$
0 \leqq M\left(x_{1}\right) \leqq M\left(x_{2}\right)
$$

since $u^{2}(x)$ is also symmetrically decreasing about $x=0$. Hence

$$
\int_{-a}^{a} p(x) u^{2}(x) d x \leqq \int_{-a}^{a} q(x) u^{2}(x) d x .
$$

The result now follows at once since by (16),

$$
\begin{aligned}
\lambda_{p} & =\frac{\int_{-a}^{a}\left[u^{\prime}(x)\right]^{2} d x}{\int_{-a}^{a} p(x) u^{2}(x) d x} \geqq \frac{\int_{-a}^{a}\left[u^{\prime}(x)\right]^{2} d x}{\int_{-a}^{a} q(x) u^{2}(x) d x} \\
& \geqq \min \frac{\int_{-a}^{a}\left[y^{\prime}(x)\right]^{2} d x}{\int_{-a}^{a} q(x) y^{2}(x) d x}=\lambda_{q} .
\end{aligned}
$$

The minimum in the fourth term of (17) is taken over all functions $y(x)$ of class $C^{1}$ on $-a \leqq x \leqq a$ which vanish at $x= \pm a$, and for which

$$
\int_{-a}^{a} q(x) y^{2}(x) d x>0 .
$$

$u(x)$ is clearly such a function, so (17) follows.

If we look on (13) and (14) as eigenvalue problems for vibrating strings (fixed at $x= \pm a$ ) with density functions $p(x)$ and $q(x)$ respectively, and having the same mass, then the theorem may be interpreted as stating that the more concentrated the mass about the centre, the lower the fundamental frequency. Cf. [2, p. 463].

\section{REFERENCES}

1. K. Tatarkiewicz, Sur une inégalité intégrale, Annales Universitatis Mariae Curie-Sklodowska, Sect. A7 (1953) (1954) pp. 83-87.

2. R. Courant and D. Hilbert, Methods of mathematical physics, vol. I, New York, 1953. 